\title{
Chewing ability and desaturation during chewing in patients with COPD
}

\author{
Takeshi Terashima, ${ }^{1}$ Takahiro Nakajima, ${ }^{1}$ Tatsu Matsuzaki, ${ }^{1}$ Eri Iwami, ${ }^{1}$ Takeo Shibui, ${ }^{2}$ Takeshi Nomura, ${ }^{2}$ \\ Akira Katakura ${ }^{3}$ \\ ${ }^{1}$ Department of Respiratory Medicine, Tokyo Dental College Ichikawa General Hospital; ${ }^{2}$ Department of Oral Medicine \\ and Maxillofacial Surgery, Tokyo Dental College Ichikawa General Hospital; ${ }^{3}$ Department of OralMaxillofacial Surgery, \\ Tokyo Dental College, Japan
}

\begin{abstract}
Chewing ability is essential to maintain nutrition status and can be associated with oral conditions, sarcopenia, and lung function in patients with chronic obstructive pulmonary disease (COPD). Herein, our pilot study investigated the chewing ability and degree of desaturation during chewing in patients with COPD $(n=41)$ and control subjects $(n=22)$. Subjects chewed a color-changing chewing gum for 1 minute and chewing ability was assessed by the color of the chewed gum, which was scored from 1 (very poor) to 5 (very good). Arterial oxygen saturation $\left(\mathrm{SpO}_{2}\right)$ was monitored using a pulse oximeter and the difference in $\mathrm{SpO}_{2}$ was determined by comparison between before and during chewing. The mean color score of the chewed gum was lower in the COPD group than in the control group $(3.1 \pm 0.7$ vs $4.2 \pm 0.9, \mathrm{p}<0.0001)$. Muscle mass loss $(p<0.05),<20$ remaining teeth $(p<0.005)$, and COPD $(p<0.001)$ were risk factors for poor chewing ability. The mean $\mathrm{SpO}_{2}$ decreased by $0.78 \pm 1.46 \%$ during gum chewing for $1 \mathrm{~min}$. The mean $\mathrm{SpO}_{2}$ during gum chewing $(95.1 \pm 2.4 \%)$ was lower than before gum
\end{abstract}

Correspondence: Takeshi Terashima, Department of Respiratory

Medicine, Tokyo Dental College Ichikawa General Hospital, 5-11-13, Sugano, Ichikawa, Chiba, 272-0824, Japan.

Tel. +81.47.322-0151; Fax: +81.47.325-4456.

E-mail: terasima@tdc.ac.jp

Keywords: Periodontitis; COPD; chewing ability; desaturation; sarcopenia.

Contributions: TT, decisions regarding patient treatment, collected clinical data, performed data analysis, wrote the manuscript; TN, TM, $\mathrm{EI}$, interpretation of clinical data; TS, TN, AK, dental examinations.

Conflict of interest: The authors declare that they have no conflict of interest.

Received for publication: 8 May 2019

Accepted for publication: 8 July 2019.

${ }^{\circ}$ Copyright: the Author(s), 2019

Licensee PAGEPress, Italy

Monaldi Archives for Chest Disease 2019; 89:1090

doi: 10.4081/monaldi.2019.1090

This article is distributed under the terms of the Creative Commons Attribution Noncommercial License (by-nc 4.0) which permits any noncommercial use, distribution, and reproduction in any medium, provided the original author(s) and source are credited. chewing $(95.9 \pm 1.7 \%)(\mathrm{p}<0.05)$. The reduction of $\mathrm{SpO}_{2}$ was greater in COPD patients who had fewer remaining teeth $(\mathrm{p}<0.05)$. COPD patients with $\mathrm{SpO}_{2}$ reduction $>4 \%$ during the 6-minute walk test showed greater reduction during gum chewing $(\mathrm{p}<0.05)$. Our results suggest that COPD patients with fewer remaining teeth exhibit poor chewing ability and greater desaturation during chewing.

\section{Introduction}

Chronic obstructive pulmonary disease (COPD) is an inflammatory disease that manifests as pulmonary dysfunction, as well as systemic comorbidities (e.g., cardiovascular diseases, osteoporosis, and skeletal muscle wasting) that worsen the patient's quality of life (QOL) [1]. Sarcopenia is common in patients with COPD, and its prevalence is associated with age, disease severity, symptoms, and comorbidity burden $[2,3]$. Sarcopenia is a known independent prognostic factor for COPD [4]. For example, the fatfree mass index was a predictor of mortality, independent of lung function among patients with COPD [5]. Muscle strength is a reliable prognostic factor of COPD [6]. Adequate nutrition is important for COPD patients [7]. It has been shown that, in COPD patients, a well-balanced diet is beneficial for pulmonary function, as well as for metabolic and cardiovascular function [8,9].

Maintaining chewing ability is essential to maintain balanced nutritional intake [10]. Sarcopenia, body mass index (BMI), and skeletal muscle mass index are associated with chewing ability and the number of remaining teeth in the aging population $[11,12]$. Periodontitis comprises a wide range of inflammatory conditions that affect the supporting structures of the teeth and can lead to tooth loss [13]. We previously reported that COPD was an independent predictive factor for periodontitis and that patients with COPD had fewer remaining teeth [14]. Therefore, we presumed that chewing ability may be impaired in patients with COPD.

COPD patients develop desaturation during walking and eating [15]. The degree of desaturation during the 6-minute walk test $(6 \mathrm{MWT})$ is associated with patient prognosis $[16,17]$. However, no study has investigated chewing ability or degree of desaturation during chewing in patients with COPD. Therefore, in this study, we examined both chewing ability and the degree of desaturation during chewing in patients with COPD.

\section{Materials and Methods}

The ethics committee of Tokyo Dental College approved the study protocol (No. 268). Patients with COPD were recruited from 
Tokyo Dental College, Ichikawa General Hospital. Current or previous smokers with a forced expiratory volume in 1 second $\left(\mathrm{FEV}_{1.0}\right)$ /forced vital capacity $<0.7$ were included in the COPD group $(n=41)$. Subjects who had malignant neoplasms within the past 3 years, as well as those who had diabetes or other serious diseases, were excluded from the study. From the same hospital, nonsmokers $(n=9)$ and smokers $(n=13)$ without COPD were selected as controls $(\mathrm{n}=22)$.

Spirometry was performed in accordance with the guidelines of the American Thoracic Society. Severity of obstruction was classified based on the guidelines of the Global Initiative for Chronic Obstructive Lung Disease [18]. The degree of dyspnea was evaluated using the modified Medical Research Council (mMRC) dyspnea scale. To assess functional status and exercise capacity, we used the patients' 6MWT distance, which comprises the distance that a patient can quickly walk along a corridor. To evaluate QOL, we used the Japanese version of the COPD assessment test (CAT), which is a questionnaire consisting of eight questions scored on a $0-5$ scale.

The body fat ratio and muscle mass were measured by bioelectrical impedance analysis using a body composition monitor (Inner Scan 50, Tanita Corporation, Tokyo, Japan). Fat-free mass was calculated by subtracting fat weight from total body weight. To determine muscle strength, handgrip strength (HGS) was measured using a grip strength dynamometer (Takei Physical Fitness Test, Niigata, Japan). A patient was diagnosed with sarcopenia when both muscle mass and strength were low. Low muscle mass was defined as values $<41.60 \mathrm{~kg}$ for men and $<31.14 \mathrm{~kg}$ for women; these were equal to or below the mean minus two standard deviations of that of healthy persons between 20 and 30 years of age in the same ethnic group [19]. In addition, low muscle strength was defined as HGS $<26 \mathrm{~kg}$ for men and $<18$ $\mathrm{kg}$ for women [20].

The number of remaining teeth was examined by trained dentists who were blinded to the patients' pulmonary function results. Chewing ability was assessed by using test chewing gum $(70 \mathrm{~mm}$ $\times 20 \mathrm{~mm} \times 1 \mathrm{~mm} ; 3.0 \mathrm{~g}$ ) that contained red, yellow, and blue dyes, as well as xylitol and citric acid (Masticatory Performance Evaluating Gum, XYLITOL, Lotte Co., Ltd., Tokyo, Japan) [21]. The red dye is $\mathrm{pH}$-sensitive and changes color under neutral or alkaline conditions. Citric acid maintains a low internal $\mathrm{pH}$ of the yellowish-green gum before chewing. During chewing, the gum changes from yellowish-green to red, as yellow and blue dyes escape into saliva and red dye appears due to citric acid elution. The subjects chewed the gum at a frequency of 1 stroke per second until they reached 60 strokes [22]. The color of the chewing gum was scored from 1 (yellow-green) to 5 (red), based on the color scale provided by the manufacturer. The color was assessed independently by two medical doctors and three dentists who were blinded to patients' lung function and oral condition and the mean of the five scores was used. Subjects with scores of 1-3 and 4-5 were assigned into a relatively low chewing ability group and a relatively high chewing ability group, respectively [10].

Data are presented as mean \pm standard deviation. The Van der Waerden test was performed to assess differences between continuous variables in the two groups. Categorical variables were compared by the $\chi^{2}$ test with Pearson's correction. Percutaneous saturation of oxygen $\left(\mathrm{SpO}_{2}\right)$ before and during gum chewing was compared using a paired $t$-test. A $\mathrm{p}$ value $<0.05$ was considered significant. Odds ratios (ORs) were estimated using both univariable and multivariable logistic regression analysis, with chewing ability as a dependent variable. Age ( $<75$ or $\geq 75$ years), muscle mass (low or normal), HGS (low or normal), number of remain- ing teeth $(<20$ or $\geq 20$ ), and presence of COPD (yes or no) were considered independent variables. All data were analyzed using JMP software for Windows, version 9.0.2 (SAS Institute Japan, Tokyo, Japan).

\section{Results}

The basic characteristics of patients with COPD and the control subjects are presented in Table 1. In the COPD group, a total of seven patients had low muscle mass, five had low HGS, and three patients had both, based on the sarcopenia criteria. In the control group, two subjects had low muscle mass, four had low HGS, and one had both. The numbers of remaining teeth were $11.5 \pm 9.0$ and $20.1 \pm 10.8$ in the COPD and control groups, respectively $(\mathrm{p}<0.005)$. The average minimal $\mathrm{SpO}_{2}$ during the 6MWT was $90.7 \pm 4.5 \%$ in the COPD group.

The mean color score of the chewing gum was lower in the COPD group than in the control group (3.1 \pm 0.7 vs $4.2 \pm 0.9$, $\mathrm{p}<0.0001)$. The proportions of subjects with a color score $\leq 3$ were $48.8 \%(20 / 41)$ and $9.1 \%(2 / 22)$ in the COPD and control groups, respectively $(\mathrm{p}<0.001)$ (Table 2$)$. Based on univariable logistic regression analysis, the unadjusted ORs for poor chewing ability (color score $\leq 3)$ were 2.04 (95\% confidence interval [CI], 0.70$6.32 ; \mathrm{p}=0.19)$ for age $\geq 75$ years $v s<75$ years; 4.75 (95\% CI 1.11 $24.8 ; \mathrm{p}<0.05)$ for low versus normal muscle mass; 0.43 (95\% CI 0.06-1.96; $\mathrm{p}=0.29)$ for low versus normal HGS; 9.39 (95\% CI $2.29-64.3 ; \mathrm{p}<0.005)$ for $<20 v s \geq 20$ remaining teeth; and 9.52 $(95 \%$ CI 2.36-64.6; $\mathrm{p}<0.001)$ for the presence of COPD vs the absence of COPD. In the multivariable regression analysis, COPD and the number of remaining teeth were significant independent factors predictive of poor chewing ability (Table 3).

The mean $\mathrm{SpO}_{2}$ decreased by $0.78 \pm 1.46 \%$ during gum chewing in the COPD group. The mean $\mathrm{SpO}_{2}$ values before and during gum chewing were $95.9 \pm 1.7 \%$ and $95.1 \pm 2.4 \%$, respectively $(\mathrm{p}<0.05)$. In the control group, the mean $\mathrm{SpO}_{2}$ decreased by $0.32 \pm 0.72 \%$ during gum chewing; the difference in $\mathrm{SpO}_{2}$ was not statistically significant. Comparisons of the reductions of $\mathrm{SpO}_{2}$ during gum chewing among COPD patients with varying numbers of remaining teeth are shown in Figure 1a. The reduction of $\mathrm{SpO}_{2}$ was greater in patients with 0-7 remaining teeth than in patients in other groups $(\mathrm{p}<0.05)$. The maximum reduction was $6 \%$ and the group with $0-7$ remaining teeth included two patients who had $\mathrm{SpO}_{2}$ reduction of at least $4 \%$. Comparisons of each variables of the COPD patients between $\mathrm{SpO}_{2}$ reduction $<2 \%(\mathrm{n}=31)$ and $\mathrm{SpO}_{2}$ reduction $\geq 2 \%$ $(n=10)$ are shown in Table 4. The mean age of patients in the $\mathrm{SpO}_{2}$ reduction $<2 \%$ group was $73.8 \pm 7.0$ years, while that in the $\mathrm{SpO}_{2}$ reduction $\geq 2 \%$ group was $78.7 \pm 5.9$ years $(p=0.055)$. COPD patients with $\mathrm{SpO}_{2}$ reduction $\geq 2 \%$ during gum chewing showed lower minimal $\mathrm{SpO}_{2}$ during the $6 \mathrm{MWT}$ than those with $\mathrm{SpO}_{2}$ reduction $<2 \%(88.0 \pm 3.7 \%$ vs $91.6 \pm 4.5 \% ; \mathrm{p}<0.05)$. The number of the remaining teeth was lower in COPD patients with $\mathrm{SpO}_{2}$ reduction $\geq 2 \%$ than in those with $\mathrm{SpO}_{2}$ reduction $<2 \%(\mathrm{p}<0.05)$. The lung function, mMRC category, CAT score, $6 \mathrm{MWT}$ distance, BMI, HGS, and serum albumin level were not significantly different between the two groups.

Figure $1 \mathrm{~b}$ shows the reduction of $\mathrm{SpO}_{2}$ during gum chewing in COPD patients with $\mathrm{SpO}_{2}$ reduction $\leq 4 \%(n=20)$ and in COPD patients with $\mathrm{SpO}_{2}$ reduction $>4 \%(n=21)$ during the $6 \mathrm{MWT}$. COPD patients with $\mathrm{SpO}_{2}$ reduction $>4 \%$ during the $6 \mathrm{MWT}$ showed greater reduction during gum chewing $(1.29 \pm 1.59 \%$ vs $0.50 \pm 0.83 \% ; \mathrm{p}<0.05)$. 


\section{Discussion}

We have shown that patients with COPD have poor chewing ability. Moreover, the number of remaining teeth and muscle mass loss were associated with decreased chewing ability. We also showed that $\mathrm{SpO}_{2}$ was reduced during 1 min of gum chewing, and that the number of remaining teeth was associated with the extent of $\mathrm{SpO}_{2}$ reduction.

Our study showed that the number of remaining teeth was a significant independent risk factor for poor chewing ability. This finding was consistent with the results of a previous study that showed an association between the number of teeth and chewing ability in elderly individuals aged $\geq 75$ years [10]. The association between muscle mass and chewing ability was also consistent with the findings of a previous study that showed an association between masticatory performance and anthropometric measurements [23]. Our study confirmed that both the volume of muscle mass and the number of remaining teeth were important factors in maintenance of chewing ability. To the best of our knowledge, this is the first study to show that COPD is an independent risk factor for poor chewing ability. Further studies are needed to elucidate the mechanism by which COPD contributes to poor chewing ability.

The small difference in $\mathrm{SpO}_{2}$ between before and during gum chewing in the COPD group could be due to normal variability of the assessment device. However, there was no difference in $\mathrm{SpO}_{2}$ between before and during gum chewing in the control group. The reduction of $\mathrm{SpO}_{2}$ was remarkable in patients who showed $\mathrm{SpO}_{2}$

Table 1. Basic characteristics of the study population.

\begin{tabular}{|c|c|c|c|}
\hline Characteristics & COPD $(n=41)$ & Control $(\mathrm{n}=22)$ & p \\
\hline Age (years) & $75.0 \pm 7.0$ & $74.6 \pm 10.0$ & 0.90 \\
\hline Sex (male/female) & $39 / 2$ & $18 / 4$ & 0.09 \\
\hline Smoking (pack-years) & $59.2 \pm 34.7$ & $20.5 \pm 26.7$ & $<0.0001$ \\
\hline Resting $\mathrm{SpO}_{2}(\%)$ & $95.9 \pm 1.7$ & $97.3 \pm 1.1$ & $<0.005$ \\
\hline $\mathrm{FEV}_{1.0}(\mathrm{~mL})$ & $1584 \pm 545$ & $2224 \pm 589$ & $<0.0005$ \\
\hline $\mathrm{FEV}_{1.0} \%$ predicted $(\%)$ & $73 \pm 22$ & $95 \pm 22$ & $<0.0005$ \\
\hline $\mathrm{FEV}_{1.0}$ forced vital capacity (\%) & $52.5 \pm 12.2$ & $72.8 \pm 8.2$ & $<0.0001$ \\
\hline GOLD COPD stage I/II/III/IV & $19 / 15 / 6 / 1$ & \multicolumn{2}{|c|}{ NA } \\
\hline mMRC dyspnea scale 0/1/2/3/4 & $15 / 18 / 5 / 3 / 0$ & \multicolumn{2}{|c|}{ NA } \\
\hline 6-minute walk test distance (m) & $383.8 \pm 93.1$ & \multicolumn{2}{|c|}{ NA } \\
\hline Minimal $\mathrm{SpO}_{2}(\%)$ during 6 -minute walk test & $90.7 \pm 4.5$ & \multicolumn{2}{|c|}{ NA } \\
\hline COPD assessment score & $11.2 \pm 8.2$ & \multicolumn{2}{|c|}{ NA } \\
\hline Serum albumin $(\mathrm{g} / \mathrm{dL})$ & $4.36 \pm 0.28$ & $4.19 \pm 0.29$ & 0.07 \\
\hline Serum CRP (mg/dL) & $0.18 \pm 0.31$ & $0.08 \pm 0.04$ & 0.59 \\
\hline Body mass index $\left(\mathrm{kg} / \mathrm{m}^{2}\right)$ & $23.4 \pm 3.1$ & $24.4 \pm 2.8$ & 0.21 \\
\hline Fat-free mass (kg) & $49.8 \pm 7.6$ & $48.9 \pm 7.4$ & 0.53 \\
\hline Muscle mass (kg) & $46.7 \pm 7.2$ & $46.3 \pm 7.0$ & 0.79 \\
\hline Hand grip strength (kg) & $32.6 \pm 7.2$ & $30.2 \pm 7.4$ & 0.17 \\
\hline Number of remaining teeth & $11.5 \pm 9.0$ & $20.1 \pm 10.8$ & $<0.005$ \\
\hline
\end{tabular}

FEV1.0, forced expiratory volume in 1 second; GOLD, Global Initiative for Chronic Obstructive Lung Disease; mMRC, modified Medical Research Council; CRP, C-reactive protein.

Table 2. Chewing ability and reduction of $\mathrm{SpO}_{2}$ during gum chewing.

\begin{tabular}{lccc} 
& COPD $(\mathbf{n = 4 1 )}$ & Control $(\mathbf{n}=22)$ & P \\
Color score & $3.1 \pm 0.7$ & $4.2 \pm 0.9$ & $<0.0001$ \\
Proportion of subjects with poor chewing ability (color score $\leq 3)$ & $48.8 \%$ & $9.1 \%$ & $<0.001$ \\
\hline Reduction of $\mathrm{SpO}_{2}(\%)$ & $0.78 \pm 1.46$ & $0.32 \pm 0.72$ & $<0.05$ \\
Proportion of subjects with $\mathrm{SpO}_{2}$ reduction $\geq 2 \%$ & $24.4 \%$ & $13.6 \%$ & 0.30 \\
\hline
\end{tabular}

Table 3. Multivariable logistic regression analysis to identify significant risk factors for poor chewing ability.

\begin{tabular}{lcc} 
& Adjusted OR (95\% CI) & P \\
Muscle mass, low $v$ s normal & $3.07(0.61-18.2)$ & 0.17 \\
Number of remaining teeth, $<20$ vs $\geq 20$ & $6.13(1.34-44.3)$ & $<0.05$ \\
\hline COPD, present/absent & $5.47(1.17-39.8)$ & $<0.05$ \\
\hline
\end{tabular}


reduction $>4 \%$ during the $6 \mathrm{MWT}$; this suggested that the reduction observed during chewing was not due to normal variability, but to reduction of the partial pressure of oxygen in the artery.

The amplitude of desaturation was relatively small and had a questionable hemodynamic consequence. A reduction of at least $4 \%$ in $\mathrm{SpO}_{2}$ has been reported to be clinically significant [24]. Although only two patients showed reduction of $\geq 4 \%$ in $\mathrm{SpO}_{2}$ during gum chewing and the mean reduction was only $0.78 \%$, the finding that COPD patients with $>4 \%$ reduction in $\mathrm{SpO}_{2}$ during the $6 \mathrm{MWT}$ showed greater reduction during gum chewing suggested that this small reduction could be indicative of a greater reduction by continued effort. The duration of 1 minute was chosen in our study because it was suitable for the assessment of chewing ability using color-changing gum. However, 1 minute could be insufficient length for estimation of oxygen uptake. We presume that a greater reduction of $\mathrm{SpO}_{2}$ could be observed with additional chew-

Figure 1. a) Reduction of $\mathrm{SpO}_{2}$ during gum chewing compared with the numbers of remaining teeth in COPD patients. Reductions in $\mathrm{SpO}_{2}$ were $1.60 \pm 0.32 \%, 0.40 \pm 0.39 \%$, and $0.50 \pm 0.35 \%$ in patients with $0-7,8-16$, and $17-28$ remaining teeth, respectively; the reduction of $\mathrm{SpO}_{2}$ in patients with 0-7 remaining teeth was greater than that in other groups $(\mathrm{p}<0.05)$. b) Reduction of $\mathrm{SpO}_{2}$ during gum chewing compared between COPD patients with $\mathrm{SpO}_{2}$ reduction $\leq 4 \%(\mathrm{n}=20)$ and $\mathrm{COPD}$ patients with $\mathrm{SpO}_{2}$ reduction $>4 \%(n=21)$ during the 6 -minute walk test; there was a greater decrease during gum chewing in the $\mathrm{SpO}_{2}$ reduction $>4 \%$ group than in the $\mathrm{SpO}_{2}$ reduction $\leq 4 \%$ group $(1.29 \pm 1.59 \%$ vs $0.50 \pm 0.83 \% ; p<0.05)$. Bars show mean \pm standard deviation.
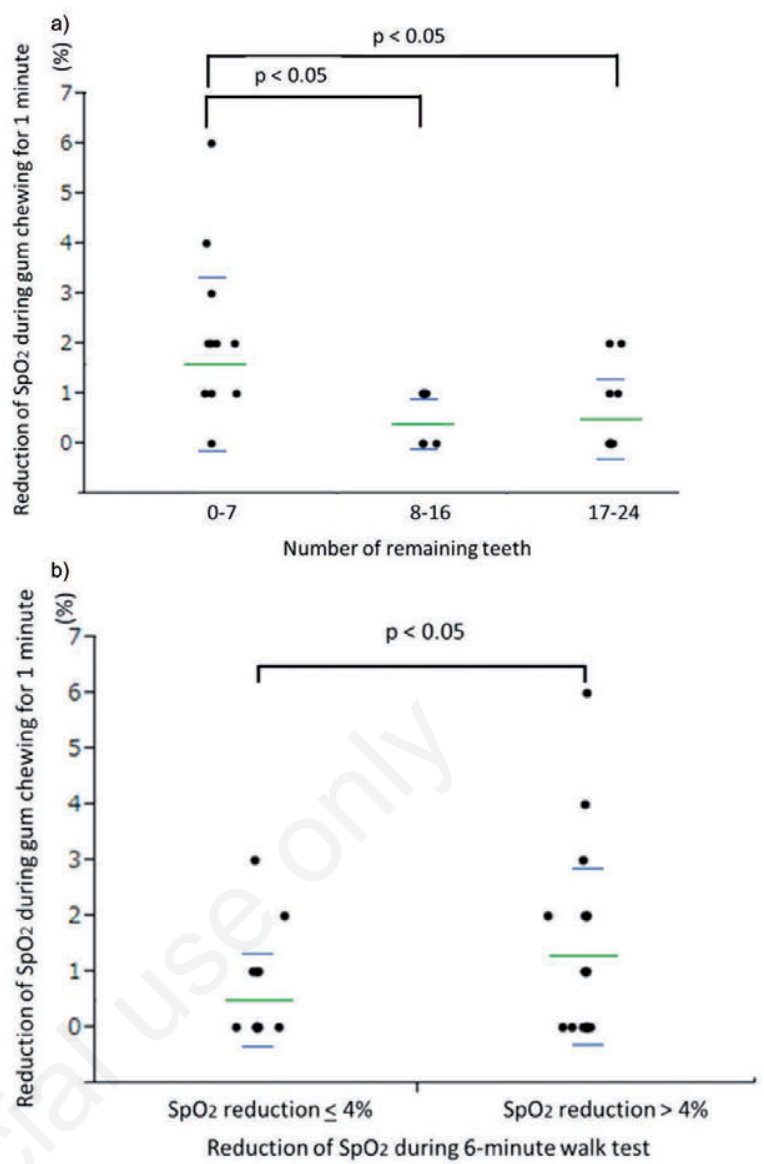

Table 4. Comparison of patient variables based on reduction of $\mathrm{SpO}_{2}$.

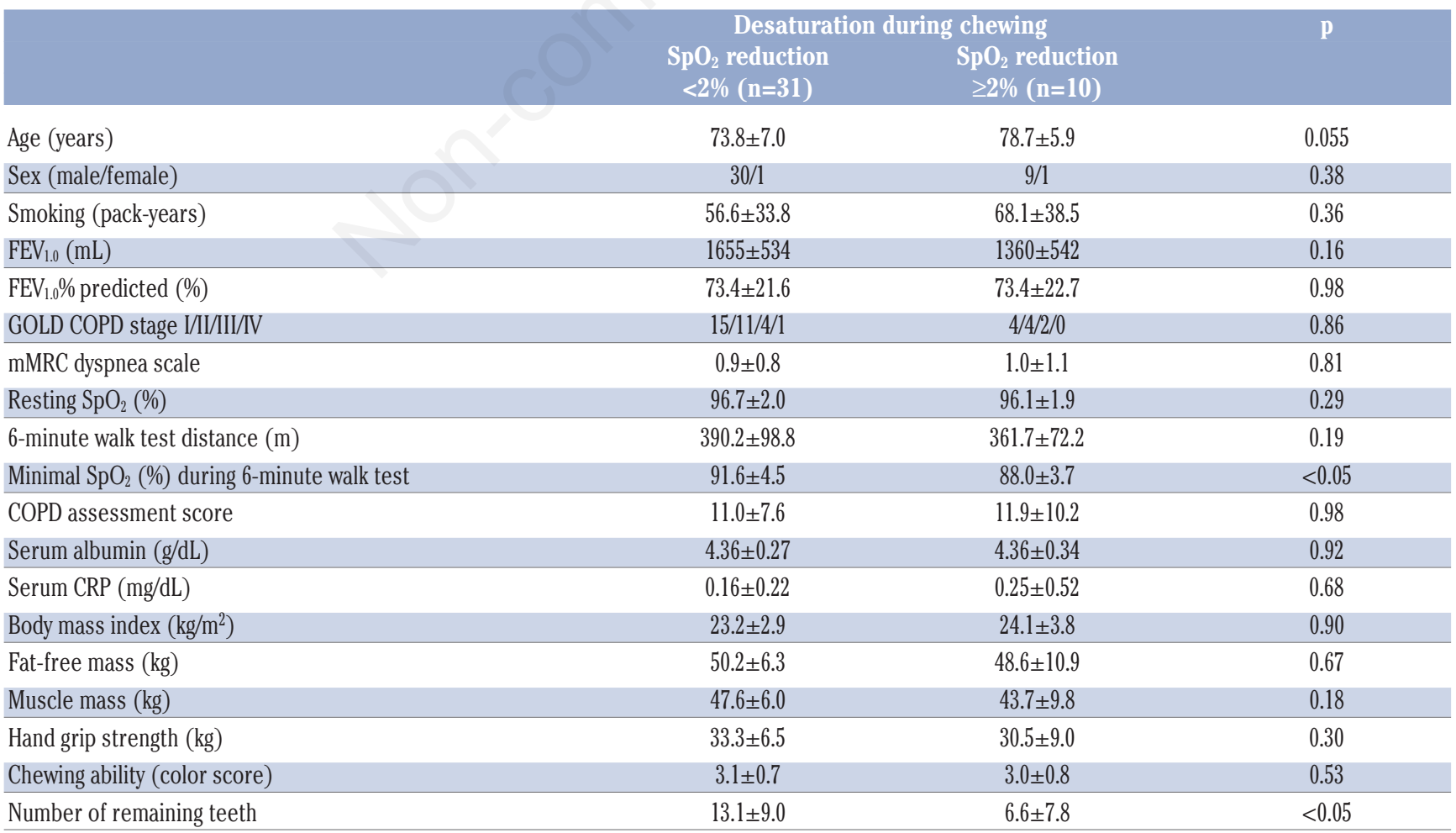

FEV1.0, forced expiratory volume in 1 second; GOLD, Global Initiative for Chronic Obstructive Lung Disease; mMRC, modified Medical Research Council; CRP, C-reactive protein. 
ing time, and tests should be performed with this additional chewing time in the future.

Desaturation during daily activities, such as walking and eating, has been shown in COPD patients $[15,25]$. Compared to a previous study that reported a mean $\mathrm{SpO}_{2}$ of $89 \%$ during eating in patients with moderate-to-severe COPD, the amplitude of desaturation was low in our study. In the previous study, desaturation began within 5 min after patients began their meals [15], whereas desaturation began within $1 \mathrm{~min}$ in the present study. This finding provides new information to aid in understanding the mechanism of desaturation during meals. Although the effort during chewing was not similar to that of walking, chewing itself could cause desaturation. Another possible mechanism may involve the metabolic effects of food absorption and digestion [25].

Surprisingly, the number of remaining teeth was associated with chewing ability and with the degree of $\mathrm{SpO}_{2}$ reduction during chewing. One possible explanation is that patients with fewer remaining teeth need more energy for gum chewing. Another explanation is that a lower number of remaining teeth contributed to irregular ventilation while chewing. As such, the preservation of natural teeth is important in patients with COPD, in order to prevent desaturation during chewing.

Our study had some limitations. The magnitude of the reduction in $\mathrm{SpO}_{2}$ induced by gum chewing for 1 min was low. Another limitation is that the power of the statistical analyses was weak because of the small number of participants. Further studies are needed with additional patients for longer test periods to support our results.

\section{Conclusions}

We have shown that chewing ability was poor and that $\mathrm{SpO}_{2}$ decreased during chewing in patients with COPD. The presence of fewer remaining teeth was associated with poor chewing ability and reduction of $\mathrm{SpO}_{2}$.

\section{References}

1. Barnes PJ, Celli BR. Systemic manifestations and comorbidities of COPD. Eur Respir J 2009;33:1165-85.

2. Jones SE, Maddocks M, Kon SSC, et al. Sarcopenia in COPD: prevalence, clinical correlates and response to pulmonary rehabilitation. Thorax 2015;70:213-8.

3. Bone AE, Hepgul N, Kon S, Maddocks M. Sarcopenia and frailty in chronic respiratory disease. Chron Respir Dis 2017; 14:85-99.

4. Steiner MC. Sarcopaenia in chronic obstructive pulmonary disease. Thorax 2007;62:101-3.

5. Schols AM, Broekhuizen R, Weling-Scheepers CA, Wouters EF. Body composition and mortality in chronic obstructive pulmonary disease. Am J Clin Nutr 2005;82:53-9.

6. Swallow EB, Reyes D, Hopkinson NS, et al. Quadriceps strength predicts mortality in patients with moderate to severe chronic obstructive pulmonary disease. Thorax 2007;62:115-20.

7. Varraso R, Camargo CA Jr. More evidence for the importance of nutritional factors in chronic obstructive pulmonary disease. Am J Clin Nutr 2012;95:1301-2.

8. Schols AM, Ferreira IM, Franssen FM, et al. Nutritional assessment and therapy in COPD: a European Respiratory Society statement. Eur Respir J 2014;44:1504-20.

9. Shalit N, Tierney A, Holland A, et al. Factors that influence dietary intake in adults with stable chronic obstructive pulmonary disease. Nutr Diet 2016;73:455-62.

10. Kimura Y, Ogawa H, Yoshihara A, et al. Evaluation of chewing ability and its relationship with activities of daily living, depression, cognitive status and food intake in the communitydwelling elderly. Geriatr Gerontol Int 2013;13:718-25.

11. Iwasaki M, Kimura Y, Ogawa H, et al. The association between dentition status and sarcopenia in Japanese adults aged $\geq 75$ years. J Oral Rehabil 2017;44:51-8.

12. Murakami M, Hirano H, Watanabe $Y$, et al. Relationship between chewing ability and sarcopenia in Japanese community-dwelling older adults. Geriatr Gerontol Int 2015;15:1007-12.

13. Kinane DF, Stathopoulou PG, Papapanou PN. Periodontal diseases. Nat Rev Disease Prim 2017;3:17038.

14. Terashima T, Chubachi S, Matsuzaki T, et al. The association between dental health and nutritional status in chronic obstructive pulmonary disease. Chron Respir Dis 2017;14:334-41.

15. Soguel Schenkel N, Burdet L, de Muralt B, Fitting JW. Oxygen saturation during daily activities in chronic obstructive pulmonary disease. Eur Respir J 1996;9:2584-9.

16. Waatevik M, Johannessen A, Gomez Real F, et al. Oxygen desaturation in 6-min walk test is a risk factor for adverse outcomes in COPD. Eur Respir J 2016;48:82-91.

17. Takigawa N, Tada A, Soda R, et al. Distance and oxygen desaturation in 6-min walk test predict prognosis in COPD patients. Respir Med 2007;101:561-7.

18. Rabe KF, Hurd S, Anzueto A, et al. Global strategy for the diagnosis, management, and prevention of chronic obstructive pulmonary disease: GOLD executive summary. Am J Respir Crit Care Med 2007;176:532-55.

19. Chen M, Sun J, Bai H, et al. Muscle mass reference standard for sarcopenia using bioelectrical impedance analysis. Asian J Gerontol Geriatr 2015;10:16-21.

20. Chen LK, Liu LK, Woo J, et al. Sarcopenia in Asia: consensus report of the Asian Working Group for Sarcopenia. J Am Med Dir Assoc 2014;15:95-101.

21. Hama Y, Kanazawa M, Minakuchi S, et al. Reliability and validity of a quantitative color scale to evaluate masticatory performance using color-changeable chewing gum. J Med Dent Sci 2014;61:1-6.

22. Kamiyama M, Kanazawa M, Fujinami Y, Minakuchi S. Validity and reliability of a self-implementable method to evaluate masticatory performance: use of color-changeable chewing gum and a color scale. J Prosthodont Res 2010;54:24-8.

23. Okada K, Enoki H, Izawa S, et al. Association between masticatory performance and anthropometric measurements and nutritional status in the elderly. Geriatr Gerontol Int 2010;10:56-63.

24. Escourrou PJ, Delaperche MF, Visseaux A. Reliability of pulse oximetry during exercise in pulmonary patients. Chest 1990;97:635-8.

25. Schols A, Mostert R, Cobben N, et al. Transcutaneous oxygen saturation and carbon dioxide tension during meals in patients with chronic obstructive pulmonary disease. Chest 1991;100: 1287-92. 
